\title{
High Performance in Low Voltage HR-STEM Applications Enabled By Fast Automatic Tuning of the Combination of a Monochromator and Probe Cs- Corrector
}

\author{
Sorin Lazar, Peter Tiemeijer, Sander Henstra, Terry Dennemans, Jan Ringnalda and Bert Freitag
}

FEI Company, P.O. Box 80066, KA 5600 Eindhoven, The Netherlands.

High tension flexibility in scanning transmission electron microscopy (STEM) enables versatility in the investigation of a broad variety of materials, which formally did not deliver the right contrast or were too beam sensitive at high acceleration voltages. By the introduction of spherical aberration (Cs) correction low voltage STEM has become the major imaging and spectroscopy technique for atomic resolution observation $[1,2]$.

When reducing the acceleration voltage and correcting the $\mathrm{Cs}$ of the objective lens, the chromatic aberration $(\mathrm{Cc})$ of the electron source becomes resolution limiting and needs to be addressed. This can be achieved by full correction or minimizing its effect on the image resolution [3]. The use of the monochromator to minimize the effect of Cc is beneficial in STEM application due to the fact that it improves the performance of EELS applications. To obtain reproducible results during daily operation the handling of a monochromized Cs corrected tool at low voltage needs simplification.

In this contribution a Wien filter monochromator [4] and a probe Cs corrector [5] combination on a cubed Titan Themis is used to maintain the atomic resolution in low voltage STEM applications. State of the art low voltage images require both the monochromator and the Cs-corrector to be tuned optimally at the same time in an easy and reproducible way. Therefore we developed fast automatic routines to tune the monochromator and the Cs corrector to give easy access to high performance in LV S/TEM. These routines are quick and deliver within minutes a completely tuned tool. We demonstrate with videos the performance of the tuning and level of automation. The automatic tuning of the monochromator requires no sample and uses the flucam of the Titan cubed as a feedback detector. For optimum Cs correction mainly the lower order aberrations vary in operation and need daily retuning. For this reason a correction routine using a series of HRSTEM images is developed to correct the lower order aberration of the Cs-corrector (focus, 2-fold astigmatism, 3-fold astigmatism, coma) This routine can run on a crystalline sample, even on the area of interest in the zone axis of the crystal to ensure best result and fastest time-to-data. An example of the automatic routine is demonstrated in figure 1, where the images of silicon [110] and GaN [211] before and after autotuning of focus and 2-fold astigmatism on the crystalline material are shown.

The negative effect of a large energy spread of the non-monochromized source is illustrated in figure 2 by looking at calculations of the transfer function in STEM with different energy resolution The calculated results are compared to images taken with different energy resolution, high tensions and beam currents, set-up via the above described automatic routines. The combination of XFEG gun with monochromator delivers even at low voltages high probe currents for analytical work. We discuss the influence of the energy resolution on the image quality. Therefore HR-STEM analytical and imaging results of various materials are presented to prove the robustness of the alignment procedures and the performance of the Wien filter/Cs corrector combination at low voltages down to 30kV. (Figure 3)

References:

[1] M Haider et al., Nature 392 (1998) 768

[2] OL Krivanek, N Dellby, and AR Lupini, Ultramicroscopy 78 (1999) 1 
[3] J Barthel, L Houben and K Tillmann, Journal of large-scale research facilities 1 (2015) A34

[4] PC Tiemeijer et al., Ultramicroscopy 114 (2012) 72

[5] H Müller et al., Microscopy and Microanalysis 12 (2006) 442
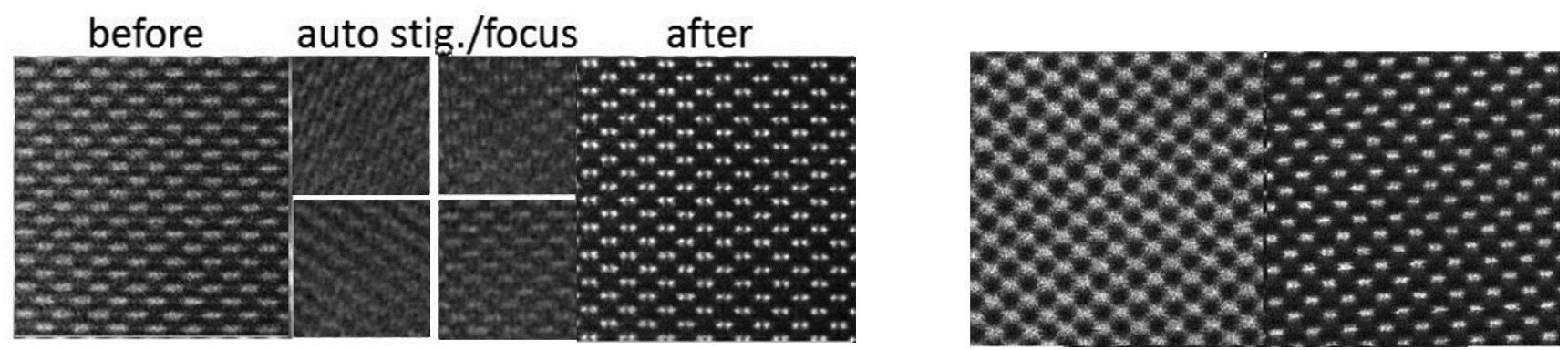

Figure1. HRSTEM images of silicon [110] (left panel) and GaN [211] (right panel) obtained before and after the automatic alignment routine of the Cs-corrector without leaving the area of interest
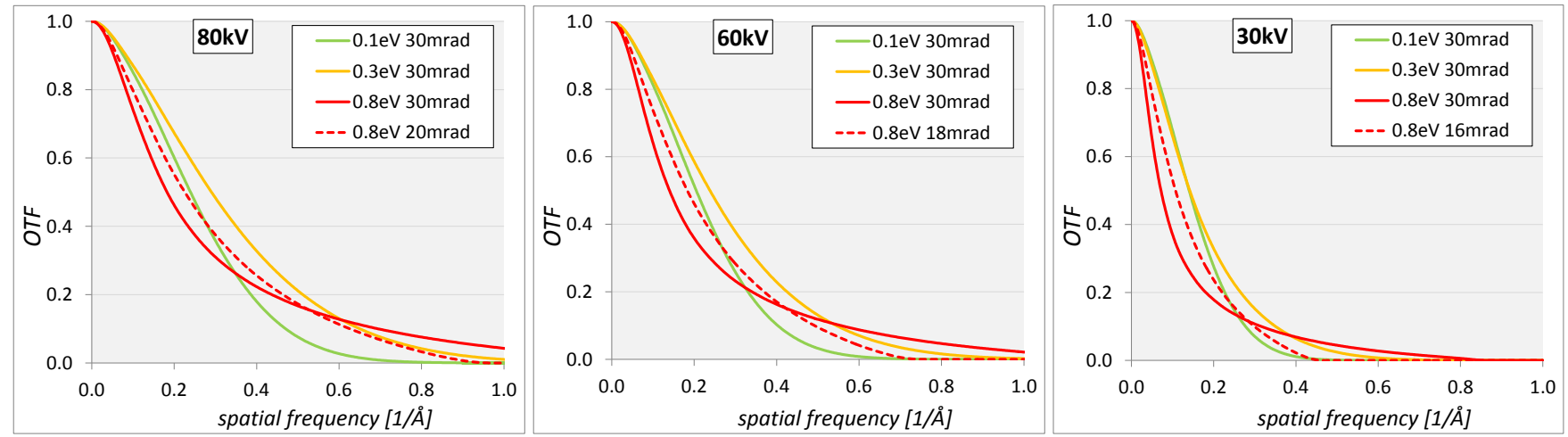

Figure 2. Optical Transfer Functions at $80 \mathrm{kV} / 60 \mathrm{kV} / 30 \mathrm{kV}$ with $\mathrm{dE}=0.1 \mathrm{eV} / 0.3 \mathrm{eV} / 0.8 \mathrm{eV}$ for $20 \mathrm{pA}$ probe current using a high-brightness Schottky X-FEG. Calculations include the effects of finite source size and of current reduction due to monochromation. The solid curves are for a fixed semi-convergence angle of 30mrad; the broken curves are for angles optimized for minimal chromatic blur. The graphs nicely show that the monochromation increases the contrast at most spatial frequencies, except at the highest frequencies where the signal drops due to the current reduction by the monochromation.
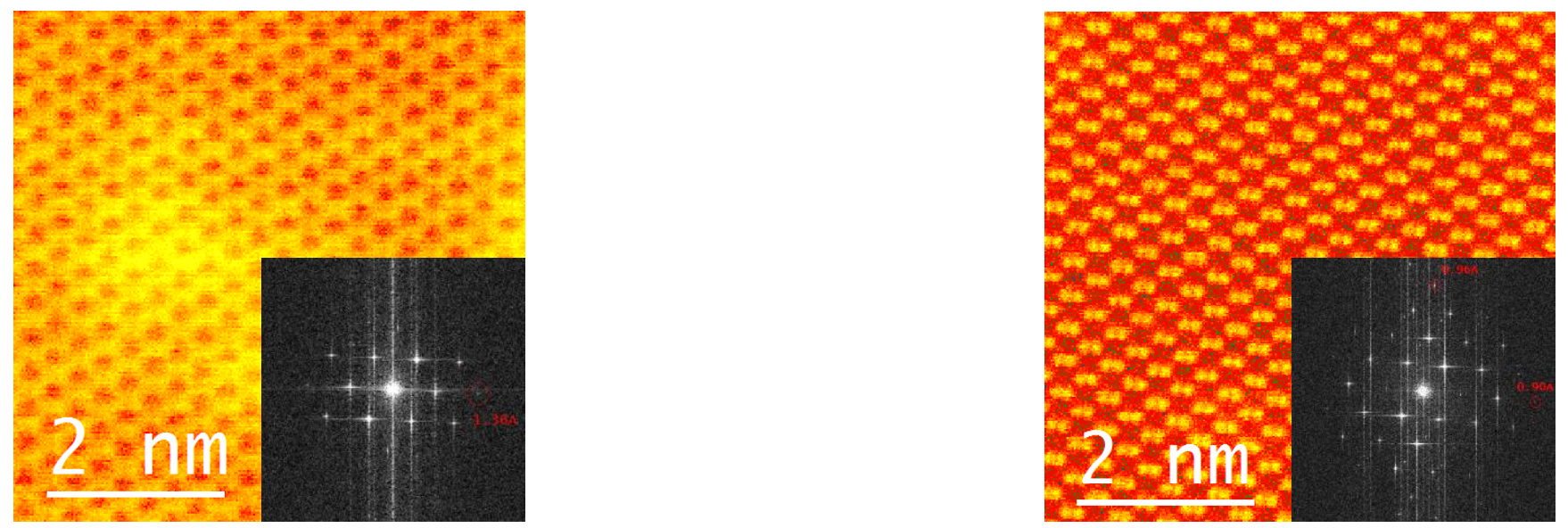

Figure 3. HR-STEM images of $\mathrm{Si}[110]$ with the monochromator excited $(\mathrm{dE}=0.21 \mathrm{eV})$. Left: at $30 \mathrm{kV}$, in the FT insert the $1.36 \AA$ reflection is visible. Right: at $80 \mathrm{kV}$; in the FT insert the $0.90 \AA$ reflection is visible 